\title{
AN EXPERT SYSTEM FOR VOLTAGE CONTROL IN A POWER SYSTEM NETWORK
}

\author{
Bansilal \\ D. Thukaram \\ K. Parthasarathy \\ Department of Electrical Engineering \\ Indian Institute of Science \\ Bangalore-560 012 INDIA \\ e-mail: dtram@vidyut.ee.iisc.ernet.in
}

\begin{abstract}
This paper presents a prototype of an expert system for alleviation of voltage violations in the day-to-day operation of large power networks. Voltage control for varying load and generation conditions can be achieved by co-ordinated control of switchable shunt VAR compensating (SVC) devices and On load transformer taps (OLTC), while generator excitations are used in dynamic control of voltage profile. This paper deals with the development of an expert system for voltage corrections for base case and contingency conditions using switchable shunt reactive compensation and transformer tap settings. The performance of the expert system is compared with the conventional optimization technique of voltage control and concurrent results have been obtained. The proposed expert system has been tested with simulated conditions of a few practical power systems. Results obtained for a 24 bus EHV Indian power network have been included for illustration purpose. The expert system is found to be suitable for on-line application in Energy Control Center as the solution is obtained very fast.
\end{abstract}

\section{INTRODUCTION}

The objective of an Energy Control Center is secure and economic operation of a power system. For the secure operation of power system it becomes essential to maintain network voltage profile within specified limits. In the day-to-day operation, power systems experience both over-voltage and under-voltage violations. These violations occur due to inadequate reactive power support for different loading conditions and network configurations. These violations can be relieved by coordinated control and switching of voltage/reactive power control devices like

- Switchable shunt VAR compensating devices.

- On load transformer taps.

- Generator excitation.

Various algorithms $[1,2,3,4]$ employing linear and non-linear optimization techniques have been reported in literature for voltage correction. These algorithms involve intensive numerical computations and hence may not be suitable in many cases for on-line application in Energy Control Centers. In Energy Control Centers it is essential to curtail the number of controllers and minimise the amount of controller movement for real time voltage/reactive power control [5]. Expert systems [6 - 11] are emerging as a Decision Support System (DSS) tool in Energy Control Centers for providing acceptable solutions for on-line applications as they mostly use symbolic processing with a minimum of numerical computations.

This paper deals with the development of an expert system for voltage corrections for base case and contingency conditions by the control of switchable shunt reactive compensation and on load transformer taps. The expert system solution methodology mainly depends on the voltage sensitivities of the load buses to different controller devices. Separate modules of the expert system have been developed. The expert system suggests voltage correction using only shunt VAR compensating devices in module 1 , using only transformer taps in module 2 and using both the shunt VAR compensating devices and the transformer taps in module 3 .

The conventional optimization technique [12] used for comparision purpose is briefly described in section 2. Sections 3,4 and 5 explain the methodology of developing the proposed expert system. Section 6 presents a summary of the results obtained for a 24 bus EHV Indian power network with discussions.

\section{OPTIMIZATION TECHNIQUE}

The optimization technique used is Least squares minimization. The objective function used is minimization of sum of the squares of voltage deviations from desired values. The control variables considered are switchable shunt reactive compensators (SVC) and on load transformer taps (OLTC). Consider a system where

$\mathrm{n}=$ total number of buses, with

$1,2, \ldots, \mathrm{g}$ generator buses $(\mathrm{g})$

$\mathrm{g}+1, \mathrm{~g}+2, \ldots, \mathrm{g}+\mathrm{s}$ SVC buses (s) and 
$\mathrm{g}+\mathrm{s}+1, \ldots, \mathrm{n}$ the remaining buses $(\mathrm{r}=\mathrm{n}-\mathrm{g}-\mathrm{s})$, and $\mathrm{t}=$ number of on load tap changing transformers.

The objective function is expressed as

$$
\min J(X)=\sum_{i=g+1}^{n}\left[V_{i}^{\text {des }}-V_{i_{(x)}^{c a l}}^{c}\right]^{2}
$$

where $\mathrm{X}$ is the vector of control variables $[X]^{t}=\left[T_{1}, \ldots, T_{t}, Q_{g+1}, \ldots, Q_{g+s}\right]$.

The condition for minimization of $\mathrm{J}(\mathrm{X})$ is $\nabla_{x} J(X)=$ 0 . Defining

$$
[H]=\left[\begin{array}{ccc}
\frac{\partial V_{g+1}}{\partial T_{1}} \ldots \frac{\partial V_{g+1}}{\partial T_{t}} & \frac{\partial V_{g+1}}{\partial Q_{g+1}} \ldots \frac{\partial V_{g+1}}{\partial Q_{g+s}} \\
\vdots & \vdots \\
\frac{\partial V_{n}}{\partial T_{1}} \ldots \frac{\partial V_{n}}{\partial T_{t}} & \frac{\partial V_{n}}{\partial Q_{g+1}} \ldots \frac{\partial V_{n}}{\partial Q_{g+s}}
\end{array}\right]
$$

We have

$$
\nabla_{x} J(X)=-2[H]^{t}\left[\begin{array}{c}
V_{g+1}^{\text {des }}-V_{g+1}^{c a l} \\
\vdots \\
V_{n}^{\text {des }}-V_{n}^{\text {cal }}
\end{array}\right]
$$

To make $\nabla_{x} J(X)$ equal zero, Newton's method is applied which gives the corrections required for the control variables as

$$
\Delta X=\left[\frac{\partial \nabla_{x} J(X)}{\partial X}\right]^{-1}\left[-\nabla_{x} J(X)\right]
$$

The jacobian of $\nabla_{x} J(X)$ is calculated by treating $H$ as a constant matrix. Hence

$$
\left[H^{t} H\right] \Delta X=[H]^{t}\left[\begin{array}{c}
V_{g+1}^{\text {des }}-V_{g+1}^{c a l} \\
\vdots \\
V_{n}^{\text {des }}-V_{n}^{\text {cal }}
\end{array}\right]
$$

\subsection{Computation of sensitivity matrix}

The elements of $\mathrm{H}$ matrix cannot be defined directly and so is evaluated as a sensitivity matrix. The relation between the net reactive power change at any bus due to change in the transformer tap setting and voltage magnitudes can be written as

$$
\left[\begin{array}{c}
\Delta Q_{g} \\
\Delta Q_{s} \\
\Delta Q_{r}
\end{array}\right]=\left[\begin{array}{cccc}
A 1 & A 2 & A 3 & A 4 \\
A 5 & A 6 & A 7 & A 8 \\
A 9 & A 10 & A 11 & A 12
\end{array}\right]\left[\begin{array}{c}
\Delta T_{t} \\
\Delta V_{g} \\
\Delta V_{s} \\
\Delta V_{r}
\end{array}\right]
$$

where

$$
\begin{aligned}
& {\left[\Delta Q_{g}\right]=\left[\Delta Q_{1}, \ldots, \Delta Q_{g}\right]^{t}} \\
& {\left[\Delta Q_{s}\right]=\left[\Delta Q_{g+1}, \ldots, \Delta Q_{g+s}\right]^{t}} \\
& {\left[\Delta Q_{r}\right]=\left[\Delta Q_{g+s+1}, \ldots, \Delta Q_{n}\right]^{t}} \\
& {\left[\Delta T_{t}\right]=\left[\Delta T_{1}, \ldots, \Delta T_{t}\right]^{t}} \\
& {\left[\Delta V_{g}\right]=\left[\Delta V_{1}, \ldots, \Delta V_{g}\right]^{t}} \\
& {\left[\Delta V_{s}\right]=\left[\Delta V_{g+1}, \ldots, \Delta V_{g+s}\right]^{t}} \\
& {\left[\Delta V_{r}\right]=\left[\Delta V_{g+s+1}, \ldots, \Delta V_{n}\right]^{t}}
\end{aligned}
$$

The sub matrices A1 to A12 are the corresponding terms of the partial derivatives $\partial Q / \partial T$ and $\partial Q / \partial V$. Transfering the control variable to the RHS and the dependent variables to the LHS we obtain

$$
\left[\begin{array}{c}
\Delta V_{s} \\
\Delta V_{r} \\
\Delta Q_{g}
\end{array}\right]=\left[\begin{array}{ll}
S 1 & S 2 \\
S 3 & S 4
\end{array}\right]\left[\begin{array}{c}
\Delta T_{t} \\
\Delta Q_{s}
\end{array}\right]
$$

where

$$
\begin{aligned}
H & =\left[\begin{array}{ll}
S 1 & S 2
\end{array}\right] \\
S 1 & =[B 1]^{-1}[B 2] \\
S 2 & =[B 1]^{-1}[B 3] \\
S 3 & =[B 4]+[-B 5][B 1]^{-1}[B 2] \\
S 4 & =[-B 5][B 1]^{-1}[B 3] \\
B 1 & =\left[\begin{array}{cc}
-A 7 & -A 8 \\
-A 11 & -A 12
\end{array}\right] \\
B 2 & =\left[\begin{array}{c}
A 5 \\
A 9
\end{array}\right] \\
B 3 & =\left[\begin{array}{c}
-I \\
0
\end{array}\right] \\
B 4 & =[A 1] \\
B 5 & =\left[\begin{array}{cc}
-A 3 & -A 4
\end{array}\right]
\end{aligned}
$$

Matrix $\mathbf{H}$ is of size $(s+r) \mathbf{x}(s+t), \mathrm{B} 1$ is of size $(s+r) \mathrm{x}(s+r), \mathrm{B} 2$ is of size $(s+r) \mathrm{x} t, \mathrm{~B} 3$ is of size $(s+r) \times s, \mathrm{~B} 4$ is of size $g \times t, \mathrm{~B} 5$ is of size $g \times(n-g)$ and $\mathrm{I}$ is an identity matrix of size $s \times s$. Matrices $\mathrm{S} 1$ and $\mathrm{S} 2$ are voltage sensitivities of load buses to transformer tap and shunt VAR compensating devices, while matrices S3 and S4 are sensitivities of generator Q to transformer tap and shunt VAR compensating devices.

\subsection{Algorithmic steps}

step 1: Read the system data.

step 2: Form network matrices.

step 3:. Perform initial power flow.

step 4: Compute the voltage error vector $\left[V^{\text {err }}\right]=\left[V^{\text {des }}-V^{\text {cal }}\right]$.

step 5: If all the voltage errors are within specified tolerance then go to step 11 .

step 6: Compute $\mathrm{H}$ matrix and $\mathrm{b}$ vector.

step 7: Solve for control variables using equation (5).

step 8: The control variables are adjusted for a suitable step size.

step 9: Control variables are updated and checked for their limits. If no scope for controller change exists then go to step 11.

step 10: Perform power flow and go to step 4 . step 11: print the results.

\section{SVC Expert System}

\subsection{Knowledge Base}

The knowledge base of the expert system consists of voltage sensitivities of load buses and generator $Q$ sensitivities to different shunt VAR compensating devices. These sensitivities are available from $\mathrm{S} 2$ and $\mathrm{S} 4 \mathrm{ma}-$ trices computed in section 2.1. The data thus obtained is arranged in the form of facts in the knowledge base of the proposed expert system. The fact consists of 2 lists, called Shunt Compensation list (SCL) for each load bus and Generator Q Shunt Sensitivity list (GQSSL) for each generator. The SCL list indicates 
in order of preference the most suitable shunt compensator for voltage correction at that load bus with the corresponding sensitivities for one step switching. For example

$\operatorname{SCL}\left(N_{i},[p, q, \ldots],\left[S_{c p}^{i}, S_{c q}^{i}, \ldots\right]\right)$.

This indicates that for node $N_{i}$, the most effective shunt compensator is located at node $\mathrm{p}$ with a sensitivity of $S_{c p}^{i}$ for one step switching, the next most effective shunt compensator is located at node $q$ with a sensitivity of $S_{c q}^{i}$ for one step switching and so on. The GQSSL list indicates the change in generator $Q$ for one step shunt compensator switching, for example

$\operatorname{GQSSL}\left(p,\left[S_{(c g)_{1}}^{p}, S_{(c g)_{2}}^{p}, \ldots, S_{(c g)_{g}}^{p}\right]\right)$.

This indicates that for one step switching of compensator located at node $p$ the change in $Q$ generation of generator 1 is $S_{(c g)_{1}}^{p}$, for generator 2 is $S_{(c g)_{2}}^{p}$ and so on. Whenever the network configuration undergoes a change the network sensitivities are recomputed.

\subsection{Expert system Rules}

The expert system is implemented in the form of a set of rules as described below.

Rule-1 If network voltage violations exist then form the following lists

1. Violation list, $\mathbf{V L}\left(V_{v 1}, V_{v 2}, \ldots\right)$, in the order of most severe violation, where $V_{v 1}$ is the most voltage violated node, $V_{v 2}$ the next most voltage violated node and so on.

2. Shunt Switching List $\operatorname{SSL}\left(\Delta C_{1}, \Delta C_{2}, \ldots, \Delta C_{s}\right)$, initialised to zero.

Rule-2 Pick a node from the top of the violation list $\left(V_{v}\right)$ and

1. choose its corresponding SCL fact from the knowledge base, i.e. $\operatorname{SCL}\left(V_{v},[p, q, \ldots],\left[S_{c p}^{v}, S_{c q}^{v}, \ldots\right]\right)$.

2. If under-voltage violation exists choose capacitive switching else if over-voltage violation exists choose inductive switching (or capacitor switch off).

3. pick the most effective compensator (e) for voltage correction from the SCL list (SCL list is arranged in order of preference).

Rule-3 Calculate the compensator switching required, $\Delta C_{e}^{r}$, for voltage correction

1. $\Delta C_{e}^{r}=\frac{V_{v}^{m i n}-V_{i}^{o}}{S_{c e}^{v}}$ for capacitive switching.

2. $\Delta C_{e}^{r}=\frac{V_{v}^{o}-V_{v}^{\max }}{S_{c e}^{v}}$ for inductive switching.

and adjust it to a suitable step size.
Rule-4 Compute the compensator (e) switching margin available, $\Delta C_{e}^{m}$, from the present setting of $C_{e}^{o}$,

1. $\Delta C_{e}^{m}=C_{e}^{\max }-C_{e}^{o}$ for capacitive switching.

2. $\Delta C_{e}^{m}=C_{e}^{o}-C_{e}^{m i n}$ for inductive switching.

3. If margin for switching is not available then pick the next most effective compensator (e) from the SCL list and go to rule 3.

Rule-5 Compare the required compensator switching $\Delta C_{e}^{r}$ with the compensator switching margin $\Delta C_{e}^{m}$ and choose the lower of the two $(\Delta C)$. If $\Delta C_{e}^{r} \geq \Delta C_{e}^{m}$ then $\Delta C=\Delta C_{e}^{m}$ else $\Delta C=\Delta C_{e}^{r}$.

Rule-6 Compute the effect of switching compensator (e) by $\Delta C$ steps on the system voltage profile using equation $V_{j}^{\prime}=V_{j}^{o}+S_{c e}^{j} \Delta C$ where $\mathrm{j}=$ $(g+1, \ldots, n)$ and check for any new voltage violations.

1. $V_{j}^{\prime}>V_{j}^{\max }$ for capacitive switching.

2. $V_{j}^{\prime}<V_{j}^{\min }$ for inductive switching.

3. If any new voltage violation occurs (say at node $\mathrm{m}$ ) then the compensator (e) switching is modified as $\Delta C=\frac{\left|V_{m}^{l i m}-V_{m}^{o}\right|}{S_{c e}^{m}}$

Rule-7 Compute the effect of change in generator $\mathrm{Q}$ value as a consequence of switching compensator (e) by $\Delta C$ steps using equation $Q_{j}^{\prime}=Q_{j}^{o}+$ $S_{(c g))_{e}}^{j} \Delta C$ where $j=1, \ldots, g$ and verify generator $\mathrm{Q}$ limit violation

1. if $Q_{j}^{\prime}>Q_{j}^{\max }$ then $\Delta C=\frac{Q_{j}^{\max }-Q_{j}^{\circ}}{S_{(\mathrm{og})_{e}}^{j}}$.

2. if $Q_{j}^{\prime}<Q_{j}^{m i n}$ then $\Delta C=\frac{Q_{j}^{\circ}-Q_{j}^{m i n}}{S_{(c g)_{e}}^{j}}$.

Rule-8 Compute the modified system voltage profile using equation $V_{j}^{\prime}=V_{j}^{o}+S_{c e}^{j} \Delta C$ and

1. update the violation list, VL.

2. update the Shunt switching list, SSL, using equation $\Delta C_{e}^{\prime}=\Delta C_{e}^{o}+\Delta C$.

Rule-9 If voltage violation at node $V_{v}$ is not completely relieved then pick the next most effective compensator from the SCL list and go to rule 3.

Rule-10 If the updated violation list is not empty then go to rule 2 .

Rule-11 The Shunt Switching List indicates the compensator, its magnitude and direction of switching for voltage correction.

\section{Transformer tap Expert system}

\subsection{Knowledge Base}

The knowledge base of the expert system consists of voltage sensitivities of load buses and generator $Q$ sensitivities to different tap changing transformers. These 
sensitivities are available from $\mathrm{S} 1$ and $\mathrm{S} 3$ matrices computed in section 2.1. The data thus obtained is stored in the form of 2 lists, called Transformer Compensation List (TCL) for each load bus and Generator Q Transformer Sensitivity list (GQTSL) for each generator. The TCL list indicates in order of preference the most effective transformer for voltage correction with corresponding sensitivities for one step tap change. For example,

TCL $\left(N_{i},\left[t_{p}, t_{q}, \ldots\right],\left[S_{t p}^{i}, S_{t_{q}}^{i}, \ldots\right]\right)$.

This indicates that for node $N_{i}$, the most effective transformer for voltage correction is $t_{p}$ with a sensitivity of $S_{t p}^{i}$ for one step tap change, the next most effective transformer is $t_{q}$ with a sensitivity of $S_{t q}^{i}$ for one step tap change and so on. The GQTSL list indicates the change in generator $Q$ for one step tap change, for example

$\operatorname{GQTSL}\left(T_{i},\left[S_{(t g)_{1}}^{i}, S_{(t g)_{2}}^{i}, \ldots, S_{(t g)_{g}}^{i}\right]\right)$

This indicates that for one step tap change at transformer $T_{i}$ the change in $\mathrm{Q}$ generation of generaror 1 is $S_{(t g)_{1}}^{i}$, for generator 2 is $S_{(t g)_{2}}^{i}$ and so on. Whenever the network configuration undergoes a change the network sensitivities are recomputed.

\subsection{Expert system rules}

The expert system is implemented in the form of a set of rules as described below:

Rule-1 If network voltage violations exist then form the following lists

1. Violation list, $\mathbf{V L}\left(V_{v 1}, V_{v 2}, \ldots\right)$, in the order of most severe violation, where $V_{v 1}$ is the most voltage violated node, $V_{v 2}$ the next most voltage violated node and so on.

2. Tap list, $\mathbf{T L}\left(\Delta T_{1}, \Delta T_{2}, \ldots, \Delta T_{t}\right)$, initialised to zero.

Check for the present tap setting of all the transformers from on-line state estimator. Pick a node from the top of the violation list $\left(V_{v}\right)$.

Rule-2 For the voltage violated node $V_{v}$

1. Select the corresponding TCL fact from the knowledge base i.e.

$\operatorname{TCL}\left(V_{v},\left[t_{p}, t_{q}, \ldots\right],\left[S_{t p}^{v}, S_{t q}^{v}, \ldots\right]\right)$.

2. Pick the most effective transformer (e) from the TCL list (TCL list is arranged in the order of preference).

Rule-3 Find the direction of tap change $\left(d_{e}\right)$ from the voltage violation direction and voltage sensitivity of the transformer.

1. If $S_{t p}^{v}>0$ then tap increase improves the voltage at $V_{v}, d_{e}=+1$

2. If $S_{t p}^{v}<0$ then tap increase decreases voltage at $V_{v}, d_{e}=-1$.
Rule-4 Check if margin for tap change $\left(\Delta T_{e}\right)$ is available in the required direction $\left(d_{e}\right)$, if not pick the next most effective transformer and go to rule 3 .

Rule-5 Compute the new system voltage profile using equation $V_{j}^{\prime}=V_{j}^{o}+S_{t e}^{j} d_{e}$ where $j=(g+1, g+$ $2, \ldots, n)$ and check for any new voltage violations $\left(V_{j}^{\min }<V_{j}^{\prime}<V_{j}^{\max }\right)$. If a new voltage violation occurs pick the next most effective transformer (e) for voltage correction and go to rule 3 .

Rule-6 Compute the effect of change in generator $Q$ value as a consequence of one step tap change at transformer (e) using equation $Q_{j}^{\prime}=Q_{j}^{o}+S_{(t g)_{e}}^{j} d_{e}$ where $j=1, \ldots, g$, and verify generator $Q$ limit violation $\left(Q_{j}^{\min }<Q_{j}^{\prime}<Q_{j}^{\max }\right)$. If a generator $\mathrm{Q}$ violation occurs then pick the next most effective transformer and go to rule 3 .

Rule-7 Compute the new system voltage profile using equation $V_{j}^{\prime}=V_{j}^{o}+S_{t e}^{j} d_{e}$ where $j=(g+1, g+$ $2, \ldots, n)$ and then update the tap list $\mathrm{TL}, \Delta T_{e}^{\prime}=$ $\Delta T_{\varepsilon}^{o}+\Delta T_{e}$ and also the violation list $\mathrm{VL}$

Rule-8 If the updated violation list is not empty then pick a node from the top of the violation list $\left(V_{v}\right)$ and go to rule 2 .

Rule-9 The Tap list TL indicates the tap changes to be carried out at different transformers for voltage correction.

\section{Combined Expert System}

In this module the expert system recommends switching action for both shunt compensation and transformer taps for voltage correction. All the facts of module I and II are combined to form the combined knowledge base. The expert system gives priority for shunt compensation switching for voltage correction. The expert system recommends switching at one most effective shunt compensator for each voltage violated node. If this is not sufficient for voltage correction then it recommends transformer tap change at the most effective transformer for each voltage violated node.

\section{Test Results}

A prototype expert system as explained above has been developed and tested with simulated conditions on a few practical power systems. The results of both the conventional optimization technique and the proposed expert system are presented for a 24 bus EHV Indian power network. The system size details are given in Table 1 and the single line diagram is as shown in fig. 1.

The input to the expert system includes the voltage violated nodes with their voltage magnitudes, present setting of the transformer taps and shunt VAR compensators, the minimum and maximum limits of the transformer taps and shunt compensating devices and 
Table 1 System data

\begin{tabular}{|l|l|}
\hline & 24 bus system \\
\hline No. of generators & 4 \\
No. of transformers & 11 \\
No. of transmission lines & 16 \\
P-generation & $2682 \mathrm{Mw}$ \\
P-load (MW) & 2620 \\
Q-load (MVAR) & 871 \\
\hline
\end{tabular}

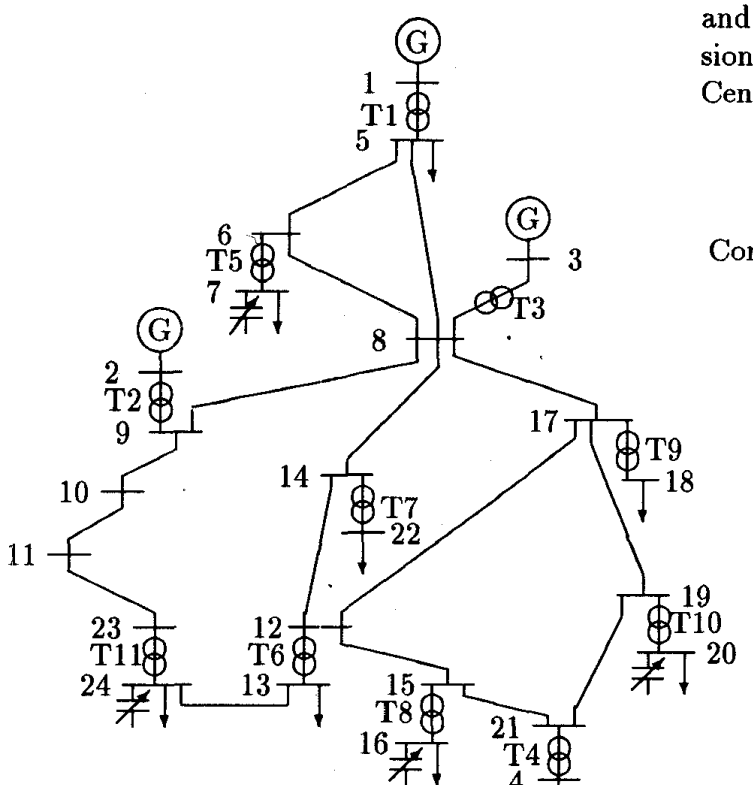

Fig.2 Single line diagram of 24 bus system

their step size. The present, minimum and maximum generator $\mathrm{Q}$ injections are also an input to the expert system to limit the controller action for any generator $Q$ violations. These inputs are assumed to be available from the on-line state estimator and network data bank.

\subsection{Discussions}

A typical set of results are presented in Tables 2 to 5 for 24 bus EHV Indian power network for a base case and contingency condition under peak load conditions. The control action and the final voltage profile as obtained for both the conventional optimization technique and expert systern are as indicated. From the results we find that the expert system performance is comparable with the conventional optimization technique. For the purpose of CPU time comparision both the methods were implemented on a PC-AT 80486 machine with $66 \mathrm{Mhz}$ clock and a math co-processor. It can be seen from the results in Table 2 and 4 that the conventional optimization technique requires between 3.15 and 3.5 secs to give a solution while the expert system provides solution in 50 msecs thus achieving a speedup of about 60 . The expert system is hence found to be suitable for real time applications.

\section{Conclusions}

A prototype of an expert system for alleviation of network voltage violations for base case and contingency conditions in the day-to-day operation has been proposed and developed. The expert system has been tested with simulated conditions of a few practical power systems and is demonstrated to give acceptable results in real time when compared to conventional optimization methods. The solution obtained is very fast and thus the expert system is found suitable as a decision support aid to the operator in the Energy Control Center.

Table 2 Base case condition

Controller settings, initial system losses $=62.59 \mathrm{Mw}$

\begin{tabular}{|c|c|c|c|}
\hline \multicolumn{4}{|c|}{ Generator excitation setting } \\
\hline 1 & \multicolumn{3}{|c|}{1.0} \\
\hline 2 & \multicolumn{3}{|c|}{1.015} \\
\hline 3 & \multicolumn{3}{|c|}{1.015} \\
\hline 4 & \multicolumn{3}{|c|}{1.015} \\
\hline \multicolumn{4}{|c|}{ Tap setting } \\
\hline Tfr & initial & ES tech & Opt. tech \\
\hline 5 & 1.000 & 0.975 & 0.9625 \\
\hline 6 & 1.000 & 0.950 & 0.9750 \\
\hline 7 & 1.000 & $1.000^{\circ}$ & 1.0000 \\
\hline 8 & 1.000 & 0.975 & 0.9875 \\
\hline 9 & 1.000 & 1.000 & 1.0000 \\
\hline 10 & 1.000 & 0.975 & 0.9750 \\
\hline 11 & 1.000 & 1.000 & 0.9750 \\
\hline \multicolumn{4}{|c|}{ SVC setting } \\
\hline node & initial & ES tech & Opt. tech \\
\hline 7 & 0.0 & 25.0 & 25.0 \\
\hline 16 & 0.0 & 20.0 & 20.0 \\
\hline 20 & 0.0 & 30.0 & 30.0 \\
\hline 24 & 0.0 & 20.0 & 20.0 \\
\hline \multicolumn{2}{|c|}{ System losses } & $60.32 \mathrm{Mw}$ & $60.33 \mathrm{Mw}$ \\
\hline \multicolumn{2}{|c|}{ CPU time (secs) } & 0.05 & 3.5 \\
\hline
\end{tabular}

Table 3 Base case condition, Voltage profile

\begin{tabular}{|c|l|l|l|}
\hline $\begin{array}{c}\text { violated } \\
\text { nodes }\end{array}$ & initial & $\begin{array}{l}\text { ES } \\
\text { tech }\end{array}$ & $\begin{array}{l}\text { Opt } \\
\text { tech }\end{array}$ \\
\hline 6 & 0.937 & 0.945 & 0.945 \\
7 & 0.895 & 0.932 & 0.945 \\
12 & 0.917 & 0.923 & 0.929 \\
13 & 0.894 & 0.948 & 0.932 \\
14 & 0.947 & 0.953 & 0.956 \\
15 & 0.930 & 0.938 & 0.942 \\
16 & 0.907 & 0.944 & 0.935 \\
18 & 0.935 & 0.943 & 0.945 \\
19 & 0.921 & 0.935 & 0.937 \\
20 & 0.894 & 0.937 & 0.940 \\
22 & 0.937 & 0.943 & 0.947 \\
23 & 0.925 & 0.972 & 0.939 \\
24 & 0.898 & 0.952 & 0.938 \\
\hline \multicolumn{4}{|l}{}
\end{tabular}


Table 4 Line (12-17) outage

Controller settings, initial system losses $=70.70 \mathrm{Mw}$

\begin{tabular}{|c|c|c|c|}
\hline \multicolumn{4}{|c|}{ Generator excitation setting } \\
\hline 1 & \multicolumn{3}{|c|}{1.025} \\
\hline 2 & \multicolumn{3}{|c|}{1.05} \\
\hline 3 & \multicolumn{3}{|c|}{1.025} \\
\hline 4 & \multicolumn{3}{|c|}{1.025} \\
\hline \multicolumn{4}{|c|}{ Tap setting } \\
\hline Tfr & initial & ES tech & Opt. tech \\
\hline 5 & 1.000 & 0.975 & 0.9750 \\
\hline 6 & 1.000 & 0.950 & 1.0000 \\
\hline 7 & 1.000 & 1.000 & 0.9875 \\
\hline 8 & 1.000 & 0.975 & 1.0000 \\
\hline 9 & 1.000 & 1.000 & 1.0000 \\
\hline 10 & 1.000 & 0.975 & 0.9875 \\
\hline 11 & 1.000 & 1.000 & 0.9500 \\
\hline \multicolumn{4}{|c|}{ SVC setting } \\
\hline node & initial & ES tech & Opt. tech \\
\hline 7 & 0.0 & 25.0 & 20.0 \\
\hline 16 & 0.0 & 20.0 & 20.0 \\
\hline 20 & 0.0 & 30.0 & 20.0 \\
\hline 24 & 0.0 & 20.0 & 20.0 \\
\hline \multicolumn{2}{|c|}{ System losses } & $67.49 \mathrm{Mw}$ & $68.72 \mathrm{Mw}$ \\
\hline \multicolumn{2}{|c|}{ CPU time (secs) } & 0.05 & 3.15 \\
\hline
\end{tabular}

Table 5 Line (12-17) outage, Voltage profile

\begin{tabular}{|c|l|l|l|}
\hline $\begin{array}{c}\text { violated } \\
\text { nodes }\end{array}$ & initial & $\begin{array}{l}\text { ES } \\
\text { tech }\end{array}$ & $\begin{array}{l}\text { Opt } \\
\text { tech }\end{array}$ \\
\hline 7 & 0.912 & 0.949 & 0.946 \\
12 & 0.860 & 0.868 & 0.881 \\
13 & 0.839 & 0.892 & 0.864 \\
14 & 0.914 & 0.922 & 0.928 \\
15 & 0.904 & 0.914 & 0.920 \\
16 & 0.882 & 0.919 & 0.902 \\
19 & 0.922 & 0.936 & 0.935 \\
20 & 0.894 & 0.939 & 0.923 \\
22 & 0.905 & 0.912 & 0.930 \\
23 & 0.879 & 0.926 & 0.863 \\
24 & 0.847 & 0.900 & 0.876 \\
\hline
\end{tabular}

\section{References}

1. H.H Hopp Optimal power dispatch - A comprehensive survey, IEEE Trans. PAS, May/June 1977.

2. J. Carpenter Optimal power flows, Electrical power and energy systems, April 1979, pp $3-15$.

3. K.Y. Lee and J.L. Ortiz Optimal real and reactive power dispatch, Electric power systems research, 1984, pp $201-212$.

4. D.S. Kirschen and H.P. Van $M W /$ Voltage control in a linear programming based power flow, IEEE Trans. PAS, May 1988.

5. S.A. Soman, K. Parthasarathy and D. Thukaram Curtailed number and reduced controller movement optimization algorithms for real time voltage/reactive power controller, IEEE/PES winter meeting 1994 New York.

6. C.C. Liu and K. Tomsovic An expert system as- sisting decision-making of reactive power/voltage control, IEEE Trans. PWRS, August 1986, pp 195-201. 7. S.J. Cheng, O.P. Malik and G.S. Hope $A n$ expert system for voltage and reactive power control of a power system, IEEE Trans. PWRS, November 1988, pp $1449-1455$.

8. Z.Z.Zhang, G.S.Hope and O.P.Malik Expert Systems in Electric Power Systems - A bibliographical survey, IEEE Trans. PWRS, October 1989, pp 1355 1362 .

9. T.F.Godart and H.B.Puttgen $A$ reactive path concept applied within a voltage control expert system, IEEE Trans. PWRS, 1991, pp 787 - 795.

10. C.C. Liu and T.S. Dillon State-of-the-art expert system applications to power systems, Electrical power and Energy systems, April/June 1992, pp 86 - 96.

11. G.E.Antonio, J.L.M.Ramos, J.L.R. Macias and Y.L.Salinas Sensitivity-based reactive power control for voltage profile improvement, IEEE Trans. PWRS, August 1993, pp $937-945$.

12. D. Thukaram, K. Parthasarathy and B.S.R. Iyengar Optimum reactive power dispatch for alleviation of over-voltage and under-voltage conditions in a large power system operation, Proc. of $15^{\text {th }}$ National systems conference, Aligarh Muslim university, India, 1991, pp $159-163$.

Bansilal received the B.E degree in Electrical Engineering from the university of Mysore in 1982, M.E in Electrical Engineering from Indian Institute of Science, Bangalore in 1988. Since 1982 he is working as a lecturer in the department of electrical engineering at the National Institute of Engineering, Mysore. Currently he is working towards $\mathrm{Ph} . \mathrm{D}$ in the Electrical Engineering department of IISc, Bangalore. His research interests include computer aided power system analysis and expert system applications to power systems.

D. Thukaram(M '90) received the B.E degree in Electrical Engineering from Osmania University, Hyderabad in 1974, M.Tech in Integrated Power Systems from Nagpur University in 1976 and Ph.D from Indian Institute of Science, Bangalore in 1986. Since 1976, he has been with IISc, where he is currently Assistant Professor in the department of Electrical Engineering. His research interests include computer aided power system analysis, reactive power optimization, parallel processing and expert system applications to power systems.

K. Parthasarathy(SM '89) received the B.Sc degree from Mysore University in 1956, D.I.I.Sc, M.E and Ph.D in Electrical Engineering from Indian Institute of Science, Bangalore in 1959, 1961 and 1967 respectively. He is currently professor at Indian Institute of Science, Bangalore. His research interests include computer aided protection, parallel processing, reactive power optimization and real-time control of power systems. 